\title{
Differential Responsiveness of Cerebellar Purkinje Neurons to GABA and Benzodiazepine Receptor Ligands in an Animal Model of Hepatic Encephalopathy
}

\author{
Anthony S. Basile, Sergio H. Gammal, ${ }^{1}$ Kevin D. Mullen, ${ }^{1 . a}$ E. Anthony Jones, ${ }^{1}$ and Phil Skolnick \\ Section on Neurobiology, Laboratory of Neuroscience, and 'Liver Diseases Section, Digestive Diseases Branch, NIDDK, \\ National Institutes of Health, Bethesda, Maryland 20892
}

The role of the GABA-benzodiazepine receptor complex in the pathogenesis of hepatic encephalopathy was investigated by recording the electrophysiological responses of single cerebellar Purkinje neurons from rabbits with hepatic encephalopathy due to galactosamine-induced fulminant hepatic failure. Both the GABAmimetic muscimol and the benzodiazepine receptor agonist flunitrazepam were 3-4 times more potent in depressing the spontaneous activity of Purkinje neurons from rabbits with hepatic encephalopathy than from control animals. Furthermore, qualitatively different responses of Purkinje neurons to benzodiazepine receptor antagonists (Ro 15-1788 and Ro 14-7437) were found in controls and rabbits with hepatic encephalopathy. These compounds markedly excited Purkinje neurons from rabbits with hepatic encephalopathy, but had either no effect (Ro 14-7437) or partially suppressed (Ro 15-1788) the spontaneous activity of neurons from control animals. In addition, incubation of Purkinje neurons from rabbits with hepatic encephalopathy with subthreshold concentrations of Ro 147437 reduced their sensitivity to muscimol, whereas treatment of control neurons with Ro 14-7437 had no effect on their sensitivity to muscimol. Finally, Purkinje neurons from hepatic encephalopathy and control rabbits displayed no difference in sensitivity to the depressant actions of the $\alpha$-adrenoceptor agonist phenylephrine.

These findings demonstrate a differential responsiveness of Purkinje neurons from an animal model of hepatic encephalopathy to ligands that interact with the GABA-benzodiazepine receptor complex. Furthermore, the observations made in this experimental model are consistent with the involvement of the GABA-benzodiazepine receptor complex in mediating hepatic encephalopathy, and provide a potential explanation for the reported efficacy of benzodiazepine receptor antagonists in ameliorating this syndrome.

Hepatic encephalopathy (HE) is a complex neuropsychiatric disorder that complicates severe acute or chronic hepatocellular failure irrespective of its etiology. The earliest signs of $\mathrm{HE}$ reflect bilateral forebrain dysfunction, with subtle changes in behavior and intellectual processes. As the syndrome progresses, ataxia

Received June 23, 1987; revised Oct. 29, 1987; accepted Nov. 27, 1987.

Correspondence should be addressed to Dr. Anthony $\mathbf{S}$. Basile, Building 8 , Room 111, Laboratory of Neuroscience, NIDDK, NIH, Bethesda, MD 20892.

a Present address: Gastroenterology Division, Cleveland Metropolitan General Hospital, 3395 Scranton Rd., Cleveland, OH 44109.

$0270-6474 / 88 / 072414-08 \$ 02.00 / 0$ and motor impairment ensue, with the eventual loss of consciousness (Conn and Liebethal, 1978; Sherlock, 1981; Hoyumpa and Schenker, 1982; Zieve, 1982). While the pathogenesis of this disorder is unknown, metabolic derangements resulting from liver failure, such as the accumulation of ammonia, mercaptans, and short-chain fatty acids have been implicated in the development of HE (Zieve, 1982). However, none of the metabolic derangements that have been studied adequately account for the known manifestations of the syndrome.

Recently, evidence has accumulated that implicates the GABA-benzodiazepine receptor complex in the clinical manifestations and pathogenesis of HE (Jones et al., 1984; Jones and Schafer, 1986). Increased densities of GABA and benzodiazepine (BZ) receptors in the CNS have been reported in several animal models of $\mathrm{HE}$, including models of fulminant hepatic failure (FHF), cirrhosis, and portal-systemic encephalopathy (Baraldi and Zeneroli, 1982, 1984a, b; Schafer et al., 1983; Pappas, 1984; Pappas and Gordon, 1986). In addition, the abnormalities of the visual evoked response (VER) waveform of 2 chemically induced animal models of HE due to FHF resemble those found in normal animals made encephalopathic by benzodiazcpines, barbiturates, or GABAmimetics (Pappas et al., 1984; Schafer et al., 1984; Bassett et al., 1987; Jones et al., 1987). These classes of drugs are believed to produce their pharmacologic effects by interacting with multiple allosteric regulatory sites on the GABA-BZ receptor (supramolecular complex; Tallman et al., 1980; Skolnick and Paul, 1982), thereby increasing chloride conductance (MacDonald and Barker, 1978; Nicoll and Wojtowicz, 1980; Schulz and MacDonald, 1980; Hamill et al., 1983). The abnormal VER waveform observed in these animal models of HE can be normalized by the administration of either $\mathrm{BZ}$ or GABA receptor antagonists (Baraldi et al., 1984a; Bassett et al., 1987; Gammal et al., 1987), further supporting the involvement of the supramolecular complex in the pathogenesis of $\mathrm{HE}$.

Despite these findings, the involvement of the supramolecular complex in the pathogenesis of HE remains controversial (see Hoyumpa, 1986, for a review). Since the VER represents the summation of the electrical activity of a large population of neurons (Chiappa and Ropper, 1982), a quantification of the responses of single neurons to pharmacologic manipulations would provide a more direct means of examining the role of the supramolecular complex in the syndrome of $H E$. This paper reports both qualitative and quantitative differences in the effects of $B Z$ and $G A B A$ receptor ligands on the spontaneous activity of Purkinje neurons from rabbits with HE due to galac- 
A

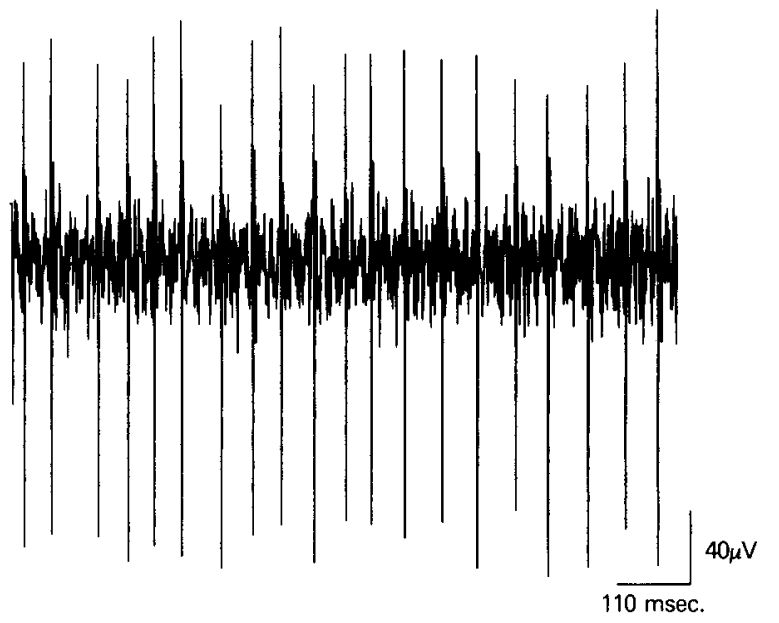

B

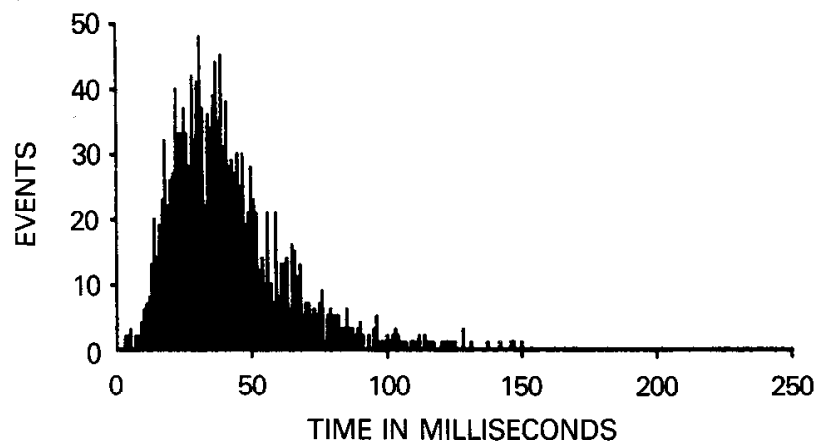

Figure 1. A, Series of action potentials from a spontaneously active Purkinje neuron recorded extracellularly from a control rabbit cerebellar slice. Approximate spike amplitude, $320 \mu \mathrm{V}$; duration, $1 \mathrm{sec}$. $B$, Interspike interval histogram of the neuronal activity in $A$. The single modal peak is indicative of an individual, regularly firing neuron. The mean firing rate of this neuron was 24.3 discharges/sec (dps), for a total of 1604 events.

tosaminc-induced FHF and control rabbits. These findings are consistent with the involvement of the supramolecular complex in an experimental model of HE.

\section{Materials and Methods}

Animals. Male New Zealand White rabbits $(\approx 2 \mathrm{~kg}$; Small Animal Section, VRB, NIH, Bethesda, MD) were maintained under a $12 \mathrm{hr}$ day/ night cycle with free access to food and water. FHF was induced using a modification of the method of Blitzer et al. (1978). Animals were infused with galactosamine $\mathrm{HCl}(4.0 \mathrm{mmol} / \mathrm{kg}$ dissolved in $8 \mathrm{ml}$ of $0.9 \%$ saline, $\mathrm{pH} 6.8$ ) over a $2 \mathrm{~min}$ period via an ear vein. Control animals received an equal volume of the vehicle. Neurologic features of encephalopathy were manifest within $12-18 \mathrm{hr}$ following galactosamine administration. The clinical stages of encephalopathy were as follows: stage I, lethargy; stage II, mild ataxia, poorly maintained head posture; stage III, severe ataxia, hindlimb extension, loss of righting reflex, nystagmus; and stage IV, coma (Bassett et al., 1987; Jones et al., 1987). Only rabbits in stages II-III of encephalopathy were studied.

Cerebellar slice preparation. Slices of cerebellar vermis were prepared using a modification of the technique for preparing rat cerebellar slices (Basile et al., 1983; Basile and Dunwiddie, 1984). Rabbits were decapitated according to AALAC guidelines, their cerebella rapidly removed and placed in ice-cold modified Ringer's medium (124 mM NaCl, $4 \mathrm{~mm}$ $\mathrm{KCl}, 3.9 \mathrm{~mm} \mathrm{MgSO}_{4}, 1.2 \mathrm{mM} \mathrm{KH}_{2} \mathrm{PO}_{4}, 0.1 \mathrm{~mm} \mathrm{CaCl}_{2}, 25 \mathrm{~mm} \mathrm{NaHCO}_{3}$, and $10 \mathrm{~mm}$ D-glucose) saturated with $95 \% \mathrm{O}_{2} / 5 \% \mathrm{CO}_{2}$. The $\mathrm{Ca}^{2+}$ and $\mathrm{Mg}^{2+}$ concentrations of this medium were altered in order to significantly inhibit ( $>90 \%$ ) evoked synaptic activity (Basile and Dunwiddie, 1984). Slices ( $400 \mu \mathrm{m}$ thick) were prepared with a Sorvall TC-2 tissue slicer and placed in an incubation chamber filled with the same medium under a humidified atmosphere of $95 \% \mathrm{O}_{2} / 5 \% \mathrm{CO}_{2}$ (Dunwiddie and Lynch, $1978)$. The slices were allowed to stabilize in this medium $\left(34^{\circ} \mathrm{C}\right)$ for at least $45 \mathrm{~min}$ before transfer to a separate recording chamber (Haas et
Table 1. Summary of neuronal responses

\begin{tabular}{|c|c|c|c|c|c|c|}
\hline & \multicolumn{3}{|c|}{ Control } & \multicolumn{3}{|l|}{$\mathrm{HE}$} \\
\hline & + & - & $\mathrm{NE}$ & + & - & $\mathrm{NE}$ \\
\hline Muscimol & & 28 & 5 & & 29 & 3 \\
\hline Flunitrazepam & 4 & 36 & 2 & 2 & 35 & 4 \\
\hline Ro $15-1788$ & & 28 & 3 & 38 & 2 & 6 \\
\hline Ro $14-7437$ & & & 25 & 31 & & 3 \\
\hline
\end{tabular}

The spontaneous activity of single Purkinje neurons was recorded from cerebellar slices derived from 19 controls and 27 rabbits with IIL. These neurons displayed a firing rate of $22.5 \pm 1.8$ and $24.9 \pm 1.6 \mathrm{dps}$ (mean \pm SEM), respectively. The table analyzes the responses of all neurons that met the criteria described in Materials and Methods. +, Excitation; -, inhibition; NE, no effect. The maximum depressions of cell firing produced by muscimol and flunitrazepam were observed at $6.9+0.3$ and $4.8 \pm 0.3 \mathrm{~min}$, respectively, after initiating superfusion. The maximum increases in firing rate of Purkinje neurons from rabbits with HE after perfusion with Ro 14-7437 and Ro 15-1788 were observed after mean latencies of $4.6 \pm 0.7$ and $2.2 \pm 0.4 \mathrm{~min}$, respectively.

al., 1979). While in the recording chamber, the cerebellar slices were continually perfused with $95 \% \mathrm{O}_{2} / 5 \% \mathrm{CO}_{2}$ saturated medium at a flow rate of $1.9-2.2 \mathrm{ml} / \mathrm{min}$. Drug solutions were added to control medium using calibrated syringe pumps (Razel Scientific Instruments) at a flow rate of $30-250 \mu \mathrm{l} / \mathrm{min}$ through a manifold in the media delivery tubing. Recordings were made from only one Purkinje neuron in each slice to minimize the possibility of drug-induced desensitization. Drugs were applied to the slice for 5-15 min intervals. Each cell was studied for 15-35 min. None of the vehicles used in this study had any effect on the spontaneous activity of Purkinje neurons when applied at flow rates up to $0.5 \mathrm{ml} / \mathrm{min}$.

Data acquisition and analysis. Action potentials were recorded extracellularly from single Purkinje neurons using glass microelectrodes (2-4 M $\Omega$ resistance) filled with $3 \mathrm{M} \mathrm{NaCl}$. The electrical activity of single Purkinje neurons was then processed and recorded electronically using standard techniques (Basile et al., 1983; Basile and Dunwiddie, 1984). Mean neuronal firing rates (in discharges per second, dps) were determined before, during, and after drug application. Neuronal responses to drugs are expressed as the ratio of the mean firing rate determined at the time of maximum drug effect to rates recorded during pre-drug control periods. If the firing rate of the neuron did not return to within $20 \%$ of the control rate after drug administration was terminated, or if an abnormal firing pattern (e.g., bursting, irregular) was observed, data from the cell were not included in the analysis. Responses from 3-6 cells were averaged and used to construct concentration-response curves. The $\mathrm{EC}_{50}$ and $\mathrm{IC}_{50}$ values of these curves were determined using probit analysis, and the $95 \%$ confidence intervals for these values determined using the Lichtfield-Wilcoxon test (Tallarida and Murray, 1987). Curves were fitted to the concentration-response data using the computer modeling program MLAB (DCRT, NIH).

Chemicals. Ro 15-1788 and flunitrazepam were dissolved in ethanol. Ro 14-7437, muscimol, and phenylephrine were dissolved in distilled water. These stock solutions were added to medium and diluted 10,000200,000 -fold during application to the slice. Ro 15-1788, Ro 14-7437, and flunitrazepam were gifts from Dr. Peter Sorter (Hoffman-LaRoche, Nutley, NJ). Galactosamine-HCl was purchased from ICN Nutritional Biochemicals (Cleveland, $\mathrm{OH}$ ), muscimol from Research Biochemicals (Wayland, MA), and phenylephrine $\mathrm{HCl}$ from Sigma (St. Louis, MO).

\section{Results}

\section{Characterization of rabbit Purkinje neurons in vitro}

Nineteen vehicle (control) and 27 galactosamine (HE)-treated rabbits were used in this study. The mean firing rates of Purkinje neurons did not differ significantly between the control and HE groups $(22.5 \pm 1.8 \mathrm{dps} ; n=131$, and $24.9 \pm 1.6 \mathrm{dps} ; n=153$, respectively), and were similar to the firing rates of Purkinje neurons from rat cerebellar slices (19.4 $\pm 0.8 \mathrm{dps}$ ) (Basile and Dunwiddie, 1984). The extracellular action potentials recorded from these neurons displayed a biphasic waveform characteristic of Purkinje neurons and exhibited a stable firing pattern (Fig. $1 A$ ). This pattern was indicated in the interspike interval 

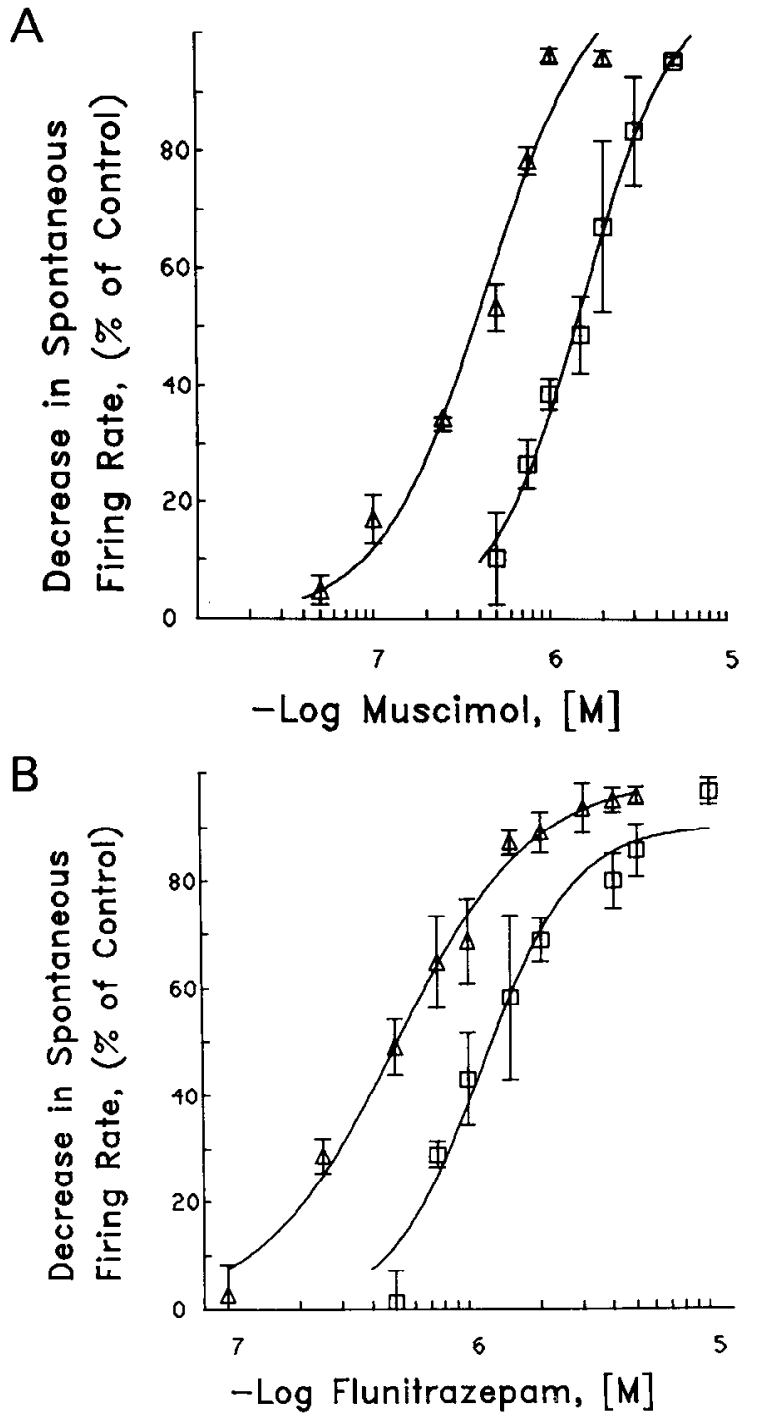

Figure 2. A, Concentration-responsc curve for the inhibition of Purkinje neuron activity by muscimol. Open triangles, HE neurons; open squares, control neurons. Each data point represents the mean \pm SEM of responses from 3-6 cells. Responses were recorded from one neuron per slice. The $\mathrm{IC}_{50}$ values of muscimol for control and HE neurons are 1.37 and $0.30 \mu \mathrm{M}$, respectively, as determined by probit analysis. $B$, Concentration-response curves for the inhibition of Purkinje neuron activity by the benzodiazepine receptor agonist flunitrazepam. Symbols as in $A$. Each data point represents the mean \pm SEM of responses from 3-5 cells. $\mathrm{IC}_{50}$ values from control and $\mathrm{HE}$ curves are 1.71 and 0.50 $\mu \mathrm{M}$, respectively, as determined by probit analysis. Curves were fitted to the data points using MLAB.

histogram by the single modal peak (Fig. $1 B$ ), which consisted of a moderately broad distribution of spike intervals relative to those observed from the rat Purkinje neuron. The identity of these neurons in the cerebellar slice was confirmed by driving them antidromically (data not shown).

\section{Responses of Purkinje neurons to GABA and benzodiazepine receptor agonists}

Superfusion of cerebellar slices with the GABA receptor agonist muscimol $(0.05-5 \mu \mathrm{M})$ produced a concentration-dependent reduction in the spontaneous activity of Purkinje neurons from both control and $\mathrm{HE}$ rabbits (Table 1). However, muscimol was $\approx 4$.2-fold more potent in depressing the spontaneous activity of neurons from rabbits with $\mathrm{HE}$ than of controls (control: $\mathrm{IC}_{50}$, 95\% confidence intervals $-1.4,0.8-2.2 \mu \mathrm{M} ; \mathrm{HE}: 0.3,0.2-0.6$ $\mu \mathrm{M})$ (Fig. $2 A$ ). A similar increase in the inhibitory potency $(\approx 3.4$ fold) of flunitrazepam $(0.10-10 \mu \mathbf{M})$ was also observed in Purkinje neurons from rabbits with $\mathrm{HE}$ (control: $\mathrm{IC}_{50}, 95 \%$ confidence intervals $-1.7,1.0-2.9 \mu \mathrm{M}$; HE: 0.5, 0.3-1.0 $\mu \mathrm{M}$ ) (Fig. $2 B$ ).

The selectivity of this differential sensitivity of Purkinje neurons to depression by GABA-BZ receptor agonists was examined using the $\alpha$-adrenoceptor agonist phenylephrine (Mueller et al., 1982; Mynlieff and Dunwiddie, 1986). No significant difference in the depression produced by phenylephrine $(50 \mu \mathrm{M})$ was observed between Purkinje neurons from controls and from rabbits with $\mathrm{HE}(37 \pm 5.6 \%$ and $40 \pm 7.9 \%$, respectively; Fig. 5).

\section{Responses of Purkinje neurons to benzodiazepine receptor antagonists}

The effects of $2 \mathrm{BZ}$ receptor antagonists, Ro 15-1788 and Ro 14-7437 (King et al., 1984; Krespan et al., 1984; Mohler and Richards, 1981) were examined in cerebellar slices from control and $\mathrm{HE}$ rabbits. In vehicle-injected rabbits, Ro 15-1788 depressed the spontaneous activity of Purkinje neurons in a concentration-dependent manner (Table 1, Fig. $3 A$ ), with an $\mathrm{IC}_{50}$ of $1.5 \mu \mathrm{M}(95 \%$ confidence interval $=0.7-3 \mu \mathrm{M})$ and a maximum inhibition of $\approx 70 \%$ (at 5 and $10 \mu \mathrm{M}$ ) (Fig. 4). This depressant action was observed in $90 \%$ of the neurons examined (Table 1). In contrast, application of Ro 15-1788 to Purkinje cells from HE rabbits excited more than $85 \%$ of the cells tested (Table 1 , Fig. $3 B$ ). The maximum increase in firing rate elicited by Ro $15-1788\left(\mathrm{IC}_{50}, 0.4 \mu \mathrm{M} ; 95 \%\right.$ confidence interval, $\left.0.1-2.5 \mu \mathrm{M}\right)$ was $\approx 45 \%$ (Fig. 4). At the highest concentration of Ro 15-1788 examined $(5 \mu \mathrm{M})$, the amplitude of the excitation was reduced.

Since an apparent qualitative difference in the action of Ro 15-1788 was observed in Purkinje neurons from controls and rabbits with $\mathrm{HE}$, we studied the effect of another $\mathrm{BZ}$ receptor antagonist, Ro 14-7437. While Ro 14-7437 had no effect on the firing rate of Purkinje ncurons from control animals (0.5-7.5 $\mu \mathrm{M}$; Table 1, Fig. 3C), this compound elicited a robust excitation in $90 \%$ of the Purkinje neurons examined from rabbits with $\mathrm{HE}$ (Fig. 3D). The maximum increase in firing rate elicited by Ro $14-7437$ was $\approx 125 \%$, with an $\mathrm{EC}_{50}$ of $1.4 \mu \mathrm{M}(95 \%$ confidence interval, $0.7-3.1 \mu \mathrm{M}$ ).

Finally, although muscimol $(0.75 \mu \mathrm{M})$ was a significantly more potent depressant of Purkinje neurons from rabbits with $\mathrm{HE}$ than from control rabbits $(F(1,3)=26.753, p<0.05$; StudentNewman-Keuls multiple range test of means), pretreatment with a subthreshold concentration of Ro 14-7437 (0.5 $\mu \mathrm{M})$ abolished this difference. Coperfusion of muscimol and Ro 14-7437 depressed neuronal cell firing in control rabbits to the same extent as did muscimol alone $(28 \pm 3.5 \%$ versus $22 \pm 5.2 \%$, respectively) (Fig. 5). In contrast, Ro 14-7437 reduced the depressant actions of muscimol on Purkinje neurons from rabbits with $\mathrm{HE}$ $(25 \pm 3.2 \%$ versus $72 \pm 6.8 \%$ in the presence and absence of Ro 14-7437, respectively) (Fig. 5) to values that were not significantly different from those observed in neurons from control animals perfused with muscimol alone.

\section{Discussion}

Despite recent evidence supporting an involvement of the GABA-BZ receptor chloride ionophore complex (supramolecular complex) in HE, this hypothesis remains controversial (Hoyumpa, 1986). Recent studies in animal models of HE due to 


\section{CONTROL}

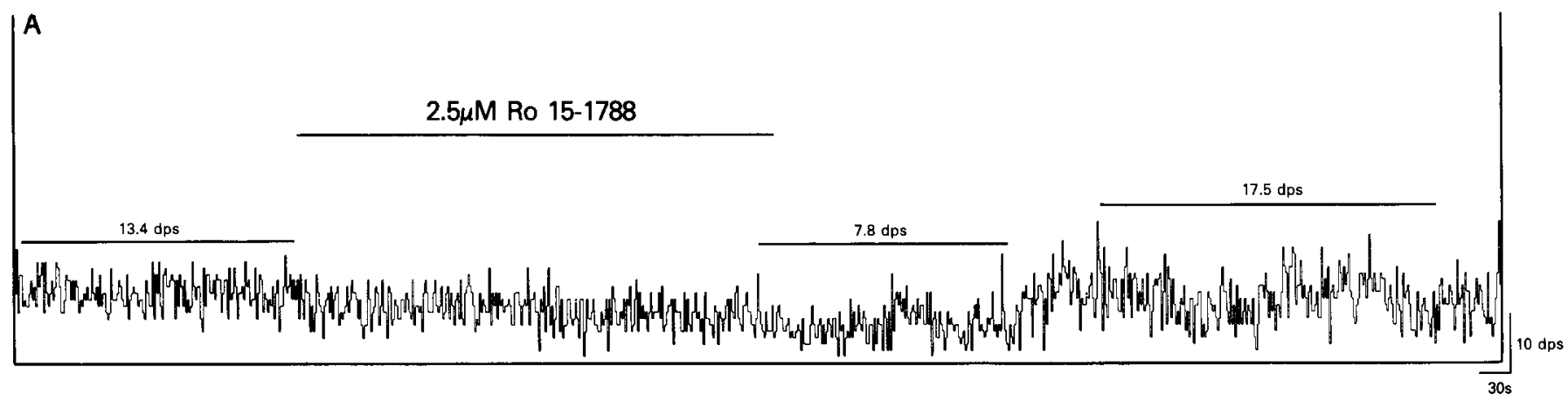

HE

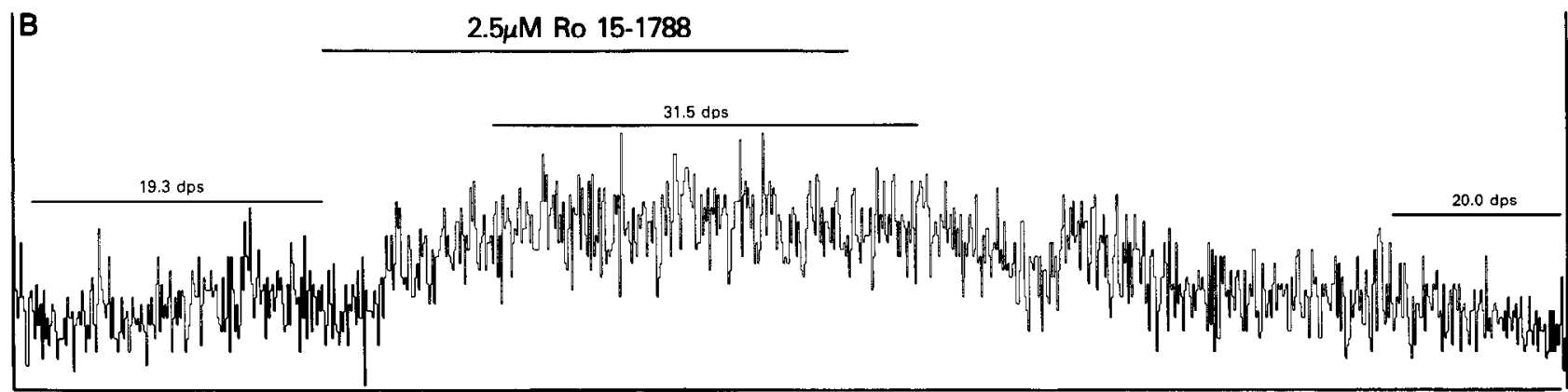

\section{CONTROL}
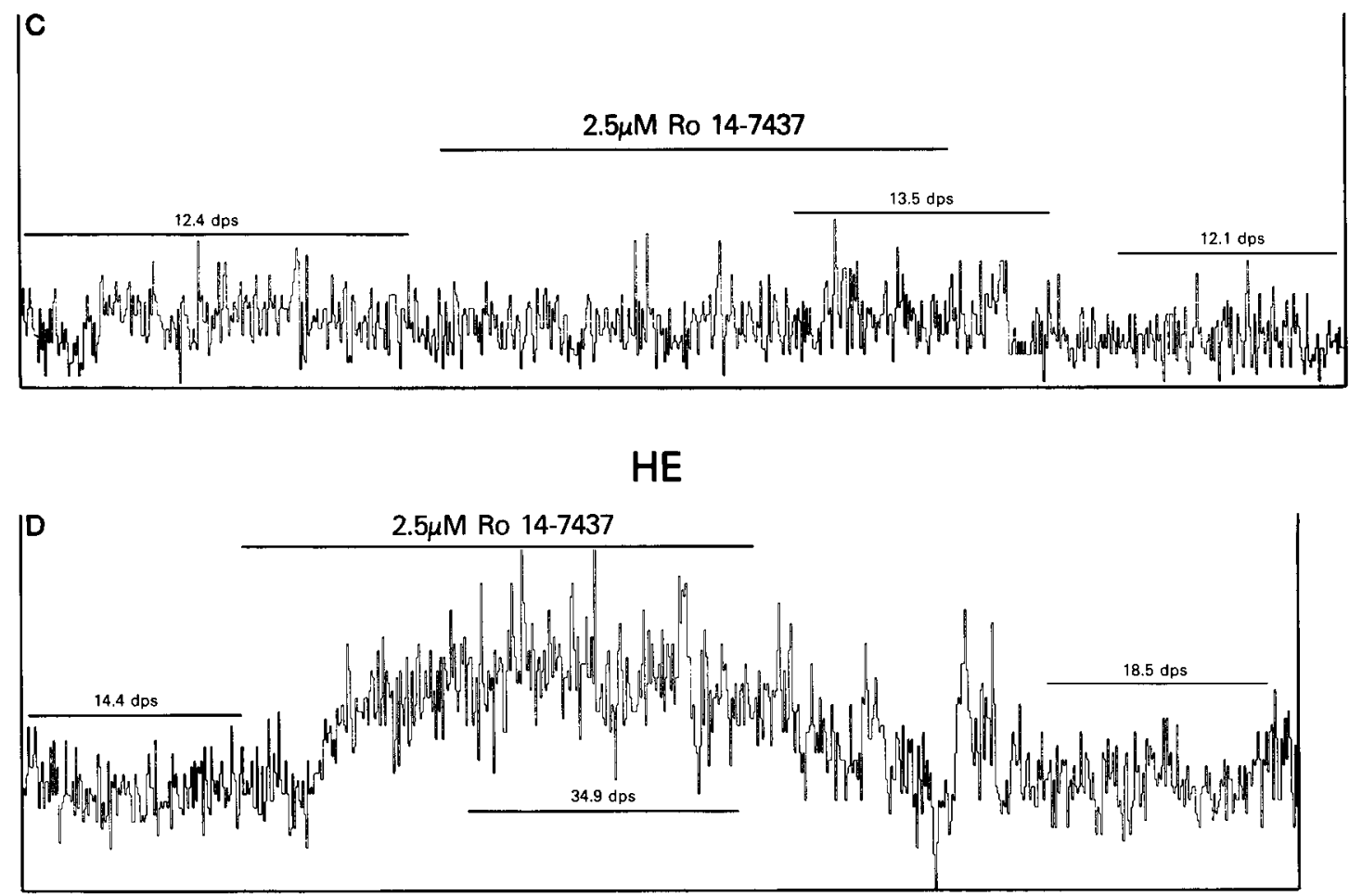

Figure 3. Ratemeter records of Purkinje neuron responses to benzodiazepine receptor antagonists. The effects of Ro $15-1788(2.5 \mu \mathrm{M})$ on the spontaneous firing rate of Purkinje neurons from controls and rabbits with HE are shown in $A$ and $B$. In these records, after a 3-5 min control period, Ro 15-1788 was applied to the slice for approximately 5 min. After a short latency, Ro $15-1788$ inhibited (42\%) the activity of control rabbit Purkinje neurons, but excited neurons from rabbits with HE (63\%). These effects were reversible, as indicated by the recovery of neuronal firing after the termination of drug application. $C$ and $D$ show the effects of Ro 14-7437 $(2.5 \mu \mathrm{M})$ on Purkinje neurons from rabbits with HE and controls. This concentration of antagonist had no significant effect on the spontaneous firing of the control rabbit Purkinje neuron $(C)$, but markedly increased the firing rate of neurons from $\mathrm{HE}$ rabbits (142\%). Lines in the lower half of $A-D$ represent the mean firing rate of the neuron in dps, averaged over the period indicated by the length of the line. Calibration bar $(A)$ applicable to all panels. 


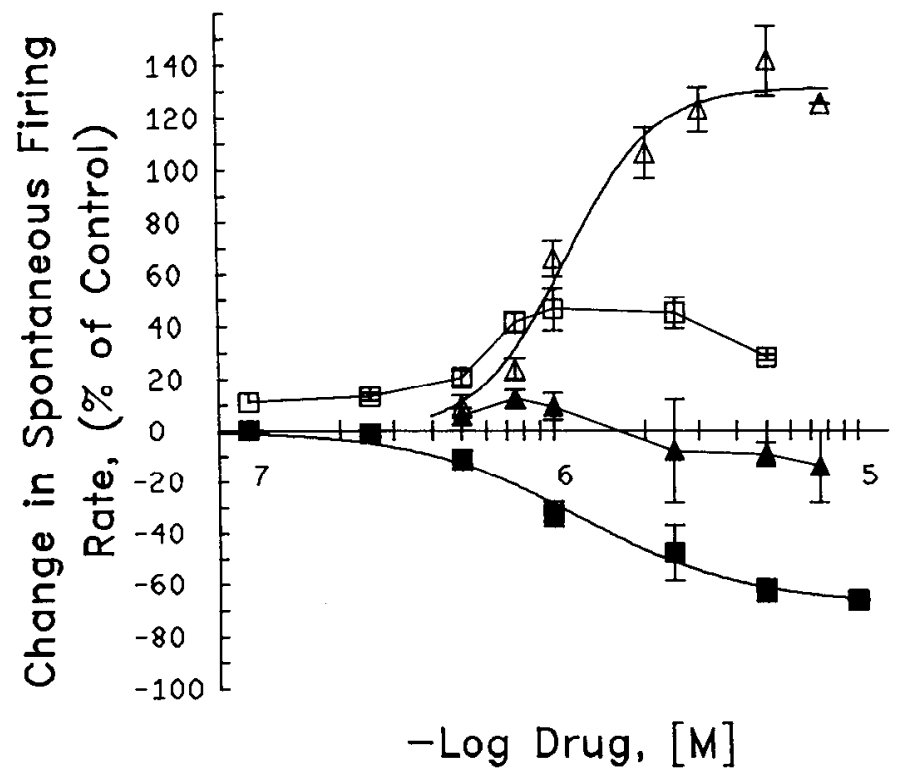

Figure 4. Concentration-response curves indicating the effects of benzodiazepine receptor antagonists on Purkinje neuron activity. Closed square, control + Ro 15-1788; closed triangle, control + Ro 14-7437; open square, HE + Ro 15-1788; open triangle, HE + Ro 14-7437. Each data point represents the mean \pm SEM of responses from 4-5 cells. As illustrated in Figure 3, Ro 15-1788 slightly decreased control Purkinje neuron spontaneous activity $\left(\mathrm{IC}_{50}-1.5 \mu \mathrm{M}\right)$. However, in Purkinje neurons from rabbits with $\mathrm{HE}$, Ro 15-1788 slightly increased the firing rate $\left(\mathrm{IC}_{50}=0.35 \mu \mathrm{M}\right)$. Note that at the highest concentration of Ro 151788 tested $(5 \mu \mathrm{M})$, the excitatory response of Purkinje neurons from rabbits with $\mathrm{HE}$ declines. In contrast, Ro 14-7437 has no effect on control rabbit Purkinje neuron activity $(0.5-7.5 \mu \mathrm{M})$ but elicits a robust increase in the spontaneous activity of Purkinje neurons from rabbits with $\mathrm{HE}\left(\mathrm{IC}_{50}=1.43 \mu \mathrm{M}\right)$. Concentration-response curves were fitted to data points using MLAB.

FHF have demonstrated that alterations in the VER during the encephalopathy resemble those observed in normal animals rendered encephalopathic by the administration of supramolccular complex ligands (such as GABAmimetics, barbiturates, or BZ receptor agonists; Schafer et al., 1984; Bassett et al., 1987; Gammal et al., 1987; Jones et al., 1987). Since the VER represents the summated electrical activity of a relatively large population of neurons (Chiappa and Ropper, 1982) and may be subject to modification by systemic metabolic changes resulting from $\mathrm{FHF}$ (Conn and Liebethal, 1978; Sherlock, 1981; Hoyumpa and Schenker, 1982; Zieve, 1982), we have investigated the electrophysiological actions of $\mathrm{BZ}$ and GABA receptor ligands on single Purkinje neurons from rabbits with experimentally induced $\mathrm{HE}$ in an attempt to elucidate further the role of this supramolecular complex in this syndrome. The cerebellar slice maintained in vitro is well-suited for such studies, since the ataxia, nystagmus, and alterations in muscle tone that occur in the rabbit model studied (Blitzer et al., 1978; Schafer et al., 1984; Bassett et al., 1987) strongly suggest the involvement of the cerebellum in this model of the syndrome. In addition, this slice preparation permits the monitoring of the responsiveness of single neurons to known concentrations of drugs in a controlled environment. Finally, the in vitro cerebellar slice technique minimizes the influence of ion and metabolite imbalances (Conn and Liebethal, 1978; Sherlock, 1981; Hoyumpa and Schenker, 1982; Zieve, 1982), peripheral drug metabolites, and alterations in bloodbrain barrier permeability (Horowitz et al., 1983; Goldstein,
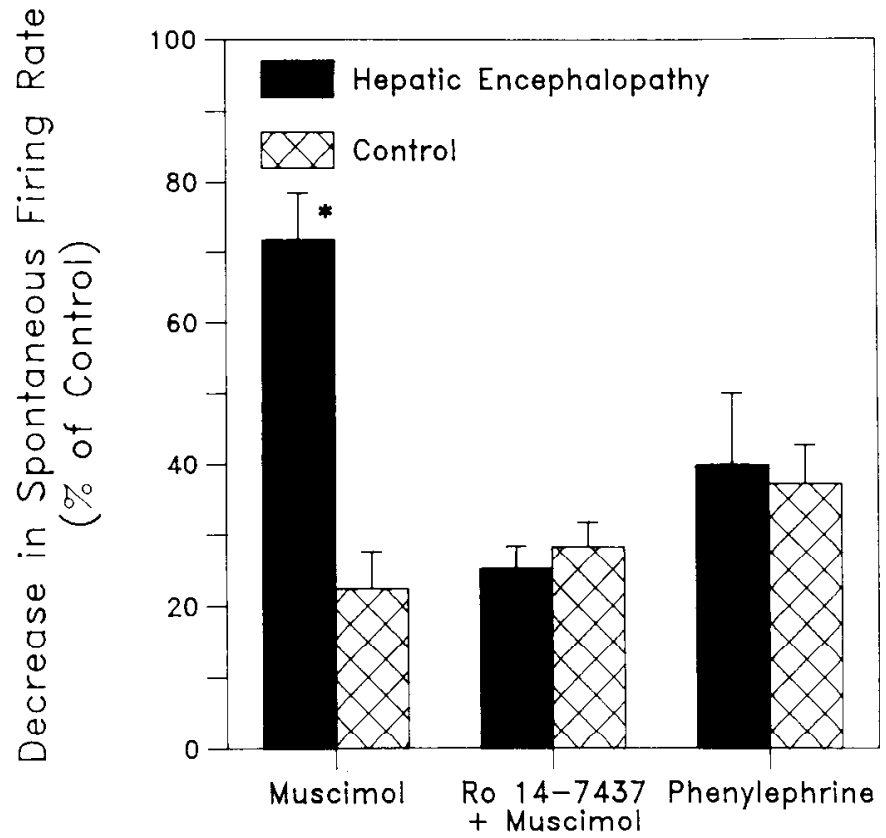

\section{Drug Treatment}

Figure 5. Bar graph illustrating the specificity of the differential sensitivity of Purkinje neurons from controls and rabbits with $\mathrm{HE}$ to depression by agents acting at the GABA-benzodiazepine receptor. Application of phenylephrine $(50 \mu \mathrm{M})$ depressed the spontaneous activity of Purkinje ncurons from controls and rabbits with $\mathrm{HE}$ to a similar extent. In addition, this bar graph illustrates the reversibility of the differential sensitivity of Purkinje neurons from controls and rabbits with $\mathrm{HE}$ inhibition by muscimol, using Ro 14-7437. Purkinje neurons from rabbits with $\mathrm{HE}$ were significantly more sensitive to depression by muscimol $(0.75 \mu \mathrm{M})$ alone than were control rabbit Purkinje neurons. However, pretreatment of Purkinje neurons from rabbits with HE, using a subthreshold concentration of Ro 14-7437 $(0.5 \mu \mathrm{M})$, reduced the subsequent muscimol-induced depression to levels indistinguishable from those occurring in control rabbit neurons. *, Amplitude of depression of Purkinje neurons from rabbits with $\mathrm{HE}$ by $0.75 \mu \mathrm{M}$ muscimol is significantly greater than that observed in control + muscimol, HE + Ro 14-7437 + muscimol, and control + Ro 14-7437 + muscimol groups (2-way ANOVA: animal model versus drug application, $F(1,3)=$ 26.753, $p<0.001 ; \mathrm{HE}+$ muscimol versus all other groups, $p<0.05$; Student-Newman-Keuls multiple range test). No significant difference in the amplitude of depression was observed between HE + Ro 14$7437+$ muscimol, control + muscimol, and control + Ro 14-7437 + muscimol groups.

1984; Schafer, 1984; Bassett et al., 1985) that would be encountered when studying neurons in situ from animals with $\mathrm{HE}$.

In Purkinje neurons substantially isolated from local and extrinsic synaptic inputs (Basile et al., 1983; Basile and Dunwiddie, 1984), the GABAmimetic muscimol and the BZ receptor agonist flunitrazepam were significantly (3.4-4.2-fold) more potent in depressing the firing rates of Purkinje neurons from rabbits with $\mathrm{HE}$ than from corresponding control animals. Furthermore, a qualitative difference was observed between the response of Purkinje neurons from control rabbits and those with $\mathrm{HE}$ to the $\mathrm{BZ}$ receptor antagonists Ro 15-1788 and Ro 147437. These compounds increased cell firing in neurons from rabbits with $\mathrm{HE}$ and depressed, or had no effect on, the firing of neurons from control rabbits. Finally, the BZ receptor antagonist Ro 14-7437 did not alter the depressant action of muscimol on neurons from control animals, but reduced the inhib- 
itory effect of muscimol on neurons from animals with $\mathrm{HE}$ to levels indistinguishable from controls.

There are several possible explanations for the differential responsiveness of Purkinje neurons from rabbits with $\mathrm{HE}$ and control animals to $\mathrm{BZ}$ and GABA receptor ligands. An increased density of both GABA and $B Z$ receptors has been reported in experimental models of HE (Baraldi and Zeneroli, 1982; Schafer et al., 1983; Baraldi et al., 1984a, b; Pappas, 1984; Pappas and Gordon, 1986). However, this increase is not universally observed, and may depend upon the method of tissue preparation (A. S. Basile and K. D. Mullen, unpublished observations). Furthermore, while such density increases (if present) could account for the increased potency of muscimol and flunitrazepam in depressing Purkinje neurons from rabbits with HE, they cannot explain the qualitative differences observed in response to $\mathrm{BZ}$ receptor antagonists.

Altered Purkinje neuron permeability or hyperpolarization due to increased concentrations of ammonia, mercaptans, or other metabolites, or decreased $\mathrm{K}^{+}$concentrations in the extracellular space as a result of liver failure, might also account for the observed electrophysiological differences (Conn and Liebethal, 1978; Sherlock, 1981; Zieve, 1981, 1982; Duffy and Plum, 1982; Hoyumpa and Schenker, 1982). It is possible that the continuous perfusion of cerebellar slices with a defined medium would partially, if not completely, correct abnormal concentrations of these substances in the slice. Furthermore, if generalized changes in neuronal polarization and permeability were present in Purkinje neurons from rabbits with HE, these cells would be more sensitive to depressants acting at loci other than the supramolecular complex. However, no differences in neuronal sensitivity to depression by the $\alpha$-adrenoceptor agonist phenylephrine were observed, suggesting an increased sensitivity of these cells to a specific class of depressants.

Several studies have reported that increased concentrations of gut-derived GABA occur in the CNS and plasma during $\mathrm{HE}$ (Ferenci et al., 1983; Bassett et al., 1985; Levy et al., 1987; van Berlo et al., 1987). While these findings are controversial, increased brain $\mathrm{GAB} \Lambda$ concentrations might explain the increased potency of flunitrazepam and muscimol in depressing Purkinje neurons from HE rabbits, but could not account for the qualitatively different responses of Purkinje neurons from controls and rabbits with $\mathrm{HE}$ to $\mathrm{BZ}$ receptor antagonists. This hypothesis is further supported by the inability of benzodiazepine receptor antagonists to affect the muscimol-induced depression of control rabbit Purkinje neurons. Finally, since stress can modulate the function of the supramolecular complex, the procedure used to induce HE may be sufficiently stressful to produce the differences observed in this study (Havoundjian et al., 1986; Schwartz et al., 1987; Trullas et al., 1987). While benzodiazepine receptor antagonists are effective in reversing the encephalopathy in some animal models of HE (the galactosamine-treated rabbit and thioacetamide-treated rat; Bassett et al., 1985; Gammal et al., 1987), they are ineffective in other proposed models of $\mathrm{HE}$, such as the portacaval-shunted rat (Rzepczynski et al., 1987). Since the procedure used to produce the latter model is probably no less stressful to the animal than that used in the galactosaminetreated rabbit, it appears that the observed changes in the function of the supramolecular complex cannot be attributed to stress.

Alternatively, long-term exposure to high levels of GABA might alter the characteristics of the supramolecular complcx (Gallager et al., 1984; Little et al., 1987), such that BZ receptor antagonists with no intrinsic actions in control animals behave as inverse agonists in rabbits with HE. At present, there is no direct evidence to support this hypothesis. Further, it has been shown that chronic treatment with BZs or exposure to GABA or GABAmimetics produce compensatory changes in function (e.g., diazcpam trcatment lowers GABA sensitivity; Gallager et al., 1984). Thus, elevated GABA levels would be expected to decrease the sensitivity of neurons to benzodiazepines and GABAmimetics. However, these effects were not observed in the present study.

The electrophysiological differences between cerebellar Purkinje neurons from control rabbits and rabbits with $\mathrm{HE}$ are most readily explained by the presence, increased production, or increased availability to neurons from $\mathrm{HE}$ rabbits of a substance that mimics the electrophysiological actions of flunitrazepam. The presence of such a compound would increase neuronal sensitivity to the depressant actions of both muscimol and flunitrazepam (Polc and Haefely, 1976; Gallager, 1978; MacDonald and Barker, 1978; Study and Barker, 1981; Hamill et al., 1983). Furthermore, displacement of this substance by Ro 15-1788 or Ro 14-7437 would disinhibit Purkinje neurons, producing excitations in these neurons and antagonizing their hypersensitivity to muscimol (Wilson and Gallager, 1987). The observation that $\mathrm{BZ}$ receptor antagonists neither increased neuronal firing nor antagonized the effect of muscimol in control Purkinje neurons suggests that the mechanisms controlling the release, uptake, synthesis, or degradation of this substance in control animals are sufficient to attentuate its actions. Alternatively, the production of this substance may be a direct consequence of liver failure.

While BZ receptor antagonists, such as Ro 15-1788 and Ro 14-7437, have no effect on normal animals in many biochemical, behavioral, and electrophysiological studies (Hunkeler et al., 1981; Mohler and Richards, 1981; Carlen et al., 1983; Skerrit and MacDonald, 1983; King et al., 1984; Krespan et al., 1984; Vicini et al., 1986), several reports have demonstrated anxiogenic and excitatory effects of these compounds (File et al., 1982; Carlen et al., 1983; Skerrit and MacDonald, 1983; King et al., 1984; Vicini et al., 1986). These effects may be attributable to the displacement of an endogenous substance with benzodiazepine-like qualities. While the identity of such a compound is unknown, several candidates (inosine, $n$-butyl- $\beta$-carboline-3carboxylate, and $N$-desmethyldiazepam) that could subserve this function have been identified in the mammalian CNS (Skolnick et al., 1978; Sangameswaran and DeBlas, 1985; Pena ct al., 1986). In addition, preliminary findings (Mullen et al., 1987; A. S. Basile, unpublished observations) indicate the presence of a substance in cerebrospinal fluid and brain extracts from rabbits with HE, but not controls, that inhibits radioligand binding to $\mathrm{BZ}$ receptors. Whether this substance is responsible for the electrophysiological effects on Purkinje neurons in rabbits with $\mathrm{HE}$ is currently under investigation.

The results of this study demonstrate an increased sensitivity of Purkinje neurons from an experimental model of HE to GABA and $\mathrm{BZ}$ receptor ligands. These findings may be related to the neuropsychiatric manifestations of $\mathrm{HE}$, and suggest that $\mathrm{BZ}$ receptor antagonists may be of therapeutic value in ameliorating HE. Recent studies have demonstrated improvements in both gross behavioral and VER abnormalities in experimental models of $\mathrm{HE}$ following $\mathrm{BZ}$ receptor antagonist administration (Baraldi et al., 1984a; Bassett et al., 1987; Gammal et al., 1987). Furthermore, initial, uncontrolled observations in humans with $\mathrm{HE}$ 
due to FHF or cirrhosis indicate that a clinical and electrophysiological remission of $\mathrm{HE}$ can be induced by Ro 15-1788 (Bansky et al., 1985; Scollo-Lavizzari and Steinmann, 1985; Grimm et al., 1987). We infer that the findings presented here have direct relevance to the mediation and amelioration of the syndrome of $\mathrm{HE}$.

\section{References}

Bansky, G., P. J. Meier, W. H. Ziegler, H. Walser, M. Schmid, and M. Huber (1985) Reversal of hepatic coma by a benzodiazepine antagonist (Ro 15-1788). Lancet 1: 1324-1325.

Baraldi, M., and M. L. Zeneroli (1982) Experimental hepatic encephalopathy: Changes in the binding of gamma-aminobutyric acid. Science 216: 427-428.

Baraldi, M., M. L. Zeneroli, E. Ventura, A. Penne, G. Pinelli, P. Ricci, and M. Santi (1984a) Supersensitivity of benzodiazepine receptors in hepatic encephalopathy due to fulminant hepatic failure in the rat: Reversal by a benzodiazepine antagonist. Clin. Sci. 67: 167-175.

Baraldi, M., M. L. Zeneroli, E. Ventura, G. Pinelli, P. Ricei, M. Santi, G. Racagni, E. Iuliano, I. Casciarri, M. Germini, E. Cavaletti, and G. Tofanetti (1984b) Portal-systemic encephalopathy in dogs: Changes in brain GABA receptors and neurochemical correlates. In Advances in Hepatic Encephalopathy and Urea Cycle Diseases, G. Kleinberger, P. Ferenci, P. Riederer, and H. Thaler, eds., pp. 353-359, Karger, Basel.

Basile, A. S., and T. V. Dunwiddie (1984) Norepinephrine elicits both excitatory and inhibitory responses from Purkinje cells in the in vitro rat cerebellar slice. Brain Res. 296: 15-25.

Basile, A. S., B. Hoffer, and T. V. Dunwiddie (1983) Differential sensitivity of cerebellar Purkinje neurons to ethanol in selectively outbred lines of mice: Maintenance in vitro independent of synaptic transmission. Brain Res. 264: 69-78.

Bassett, M. L., K. D. Mullen, B. Scholz, and E. A. Jones (1985) GABA and benzodiazepine receptor antagonists ameliorate hepatic encephalopathy in a rabbit model of fulminant hepatic failure. Hepatology 5: 1032.

Bassett, M. L., K. D. Mullen, P. Skolnick, and E. A. Jones (1987) Amelioration of hepatic encephalopathy by pharmacologic antagonism of the GABA -benzodiazepine receptor complex in a rabbit model of fulminant hepatic failure. Gastroenterology 93: 1069-1077.

Blitzer, B. L., J. G. Waggoner, E. A. Jones, H. Gralnick, O. Towne, J. Butler, V. Weise, I. Kopin, I. Walters, P. F. Teychenne, D. G. Goodman, and P. Berk (1978) A model of fulminant hepatic failure in the rabbit. Gastroenterology 74: 664-671.

Carlen, P. L., N. Gurevich, and P. Polc (1983) The excitatory effects of the specific benzodiazepine antagonist Ro 14-7437, measured intracellularly in hippocampal CAl cells. Brain Res. 271: 115-119.

Chiappa, K. H., and A. H. Ropper (1982) Evoked potentials in clinical medicine. N. Engl. J. Med. 306: 1140-1147.

Conn, H. O., and M. M. Liebethal (1978) The Hepatic Corna Syndromes, pp. 1-112, Williams and Wilkins, Baltimore, MD

Duffy, T. E., and F. Plum (1982) Hepatic encephalopathy. In The Liver: Biology and Pathobiology, I. M. Arias, H. Popper, and D. Schacter, eds., pp. 693-715, Raven, New York.

Dunwiddie, T. V., and G. Lynch (1978) Long-term potentiation and depression of synaptic responses in the rat hippocampus: Localization and frequency dependency. J. Physiol. (Lond.) 276: 353-367.

Ferenci, P., D. Covell, D. F. Schafer, J. G. Waggoner, R. Shrager, and E. A. Jones (1983) Metabolism of the inhibitory neurotransmitter GABA in a rabbit model of fulminant hepatic failure. Hepatology 3: 507-512.

File, S. E., R. G. Lister, and D. J. Nutt (1982) The anxiogenic action of benzodiazepine antagonists. Neuropharmacology 21: 1033-1037.

Gallager, D. W. (1978) Benzodiazepines: Potentiation of a GABA inhibitory response in the dorsal raphe nucleus. Eur. J. Pharmacol. 49: $133-143$.

Gallager, D. W., J. M. Lakoski, S. F. Gonsalves, and S. L. Rauch (1984) Chronic benzodiazepine treatment decreases postsynaptic GABA sensitivity. Nature 308: 5954.

Gammal, S. H., D. Geller, P. Skolnick, S. M. Paul. M. Lisker-Melman, and E. A. Jones (1987) Unequivocal amelioration of hepatic encephalopathy by benzodiazepine receptor antagonists in a rat model of fulminant hepatic failure. J. Hepatol. (Suppl. 1) 4: S17.
Goldstein, G. W. (1984) The role of brain capillaries in the pathogenesis of hepatic encephalopathy. Hepatology 4: 565-567.

Grimm, G., K. Lenz, G. Kleinberger, A. Laggner, W. Druml, B. Schneeweiss, F. Gremmel, and F. Holzner (1987) Ro 15-1788 improves coma in 4 out of 5 patients with fulminant hepatic failure; verification by long latency auditory and somatosensory evoked potentials. J. Hepatol. (Suppl. 1) 4: S21.

Haas, H. L., B. Schaerer, and M. Vosmansky (1979) A simple perfusion chamber for the study of nervous tissue slices in vitro. J. Neurosci. Methods $1:$ 323-325.

Hamill, O. P., J. Bormann, and B. Sakmann (1983) Activation of multiple-conductance state chloride channels in spinal neurons by glycine and GABA. Nature 305: 805-808.

Havoundjian, H., S. M. Paul, and P. Skolnick (1986) Rapid, stressinduced modification of the benzodiazepine receptor coupled chloride ionophore. Brain Res. 375: 401-406.

Horowitz, M. E., D. F. Schafer, P. Molnar, E. A. Jones, R. G. Blasberg, C. S. Patlak, J. Waggoner, and J. D. Fenstermacher (1983) Increased blood-brain transfer in a rabbit model of active liver failure. Gastroenterology 84: 1003-1011.

Hoyumpa, A. M. (1986) The unfolding GABA story. Hepatology 6 : $1042-1044$.

Hoyumpa, A. M., and S. Schenker (1982) Perspectives in hepatic encephalopathy. J. Lab. Clin. Med. 100: 477-487.

Hunkeler, W., H. Mohler, L. Pieri, P. Polc, E. P. Bonnetti, R. Cumin, R. Schaffner, and W. Haefely (1981) Selective antagonists of benzodiazepines. Nature 290: 514-516.

Jones, D. B., K. D. Mullen, M. Roessle, T. Maynard, and E. A. Jones (1987) Hepatic encephalopathy: Application of visual evoked responses to test hypothesis of its pathogenesis in rats. J. Hepatol. 4: $118-126$.

Jones, F. A., and D. F. Schafer (1986) Hepatic encephalopathy: A neurochemical disorder. In Progress in Liver Diseases, vol. 8, $\mathrm{H}$. Popper and F. Schaffner, eds., pp. 525-540, Grune \& Stratton, New York.

Jones, E. A., D. F. Schafer, and P. Ferenci (1984) The neurobiology of hepatic encephalopathy. Hepatology 4: 1235-1242.

King, G. L., J. J. Knox, and R. Dingledine (1984) Reduction of inhibition by a benzodiazepine antagonist, Ro 15-1788, in the rat hippocampal slice. Neuroscience 15: 371-378.

Krespan, B., S. A. Springtield, H. Haas, and H. M. Geller (1984) Electrophysiological studies on benzodiazepine antagonists. Brain Res. 295: 265-274.

Levy, L. J., J. Leek, and M. S. Losowsky (1987) Evidence for gamma amino butyric acid as the inhibitor of gamma amino butyric acid binding in the plasma of humans with liver disease and hepatic encephalopathy. Clin. Sci. 73: 531-534.

Little, H. J., D. J. Nutt, and S. C. Taylor (1987) Selective changes in the in vivo effects of benzodiazepine receptor ligands after chemical kindling with FG7142. Neuropharmacology 26: 25-33.

MacDonald, R. L., and J. L. Barker (1978) Enhancement of GABAmediated postsynaptic inhibition in cultured mammalian spinal cord neurons: A common mode of anticonvulsant action. Nature 271:563564.

Mohler, H., and J. G. Richards (1981) Agonist and antagonist benzodiazepine receptor interactions in vitro. Nature 294: 763-765.

Mueller, A. L., M. R. Palmer, B. J. Hoffer, and T. V. Dunwiddie (1982) Hippocampal noradrenergic responses in vivo and in vitro: Characterization of alpha and beta components. Naunyn Schmiedebergs Arch. Pharmacol. 318: 259-266.

Mullen, K. D., J. V. Martin, W. B. Mendelson, and E. A. Jones (1987) Further evidence that hepatic encephalopathy in the galactosamine rabbit model may be mediated by an endogenous benzodiazepine compound. J. Hepatol. (Suppl. 1) 4: S35.

Mynlieff, M., and T. V. Dunwiddie (1986) Noradrenergic responses in rat hippocampus: Evidence for inhibitory responses mediated by $\alpha_{1}$-receptors. Soc. Neurosci. Abstr. 12: 377.8

Nicoll, R., and J. Wojtowicz (1980) The effects of pentobarbital and related compounds on frog motoneurons. Brain Res. 191: 225-237.

Pappas, S. C. (1984) Increased GABA receptors in the brain precedes hepatic encephalopathy. Hepatology 4: 1051.

Pappas, S. C., and V. P. Gordon (1986) Altered brain GABA binding and synaptic plasma membrane composition in cirrhosis: $\mathrm{A}$ role in the pathogenesis of chronic hepatic encephalopathy? Hepatology 6: 1106 
Pappas, S. C., P. Ferenci, D. F. Schafer, and E. A. Jones (1984) Visual evoked potentials in a rabbit model of hepatic encephalopathy. II. Comparison of hyperammonemic encephalopathy, postictal coma, and coma induced by synergistic neurotoxins. Gastrocnterology 86: 546-551.

Pena, C., J. H. Medina, M. L. Novas, A. C. Paladini, and E. DeRobertis (1986) Isolation and identification in bovine cerebral cortex of $n$-butyl- $\beta$-carboline-3-carboxylate, a potent benzodiazepine binding inhibitor. Proc. Natl. Acad. Sci. USA 83: 4952-4956.

Polc, P., and W. Haefely (1976) The effects of two benzodiazepines, phenobarbital, and baclofen, on synaptic transmission in the cat cuneate nucleus. Naunyn Schmiedebergs Arch. Pharmacol. 294: 121131.

Rzepczynski, D., L. Zieve, S. Lindblad, and D. LaFontaine (1987) In vivo studies of GABAergic effects in experimental hepatic encephalopathy. Hepatology 6: 902-905.

Sangameswaran, L., and A. L. DeBlas (1985) Demonstration of benzodiazepine-like molecules in the mammalian brain with a monoclonal antibody to benzodiazepines. Proc. Natl. Acad. Sci. USA 82 . 5560-5564.

Schafer, D. F. (1984) The blood-brain barrier in acute liver failure. In Advances in Hepatic Encephalopathy and Urea Cycle Diseases, G. Kleinberger, P. Ferenci, P. Riederer, and H. Thaler, eds., pp. 240243, Karger, Basel.

Schafer, D. F., J. M. Fowler, P. J. Munson, A. Thakur, J. G. Waggoner, and E. A. Jones (1983) Gamma-aminobutyric acid and benzodiazepine receptors in an animal model of fulminant hepatic failure. $J$. Lab. Clin. Med. 102: 870-880.

Schafer, D. F., S. C. Pappas, I. F. Brody, R. Jacobs, and E. A. Jones (1984) Visual evoked potentials in a rabbit model of hepatic encephalopathy. I. Sequential changes and comparisons with drug-induced comas. Gastroenterology 86: 540-545.

Schulz, W., and R. McDonald (1980) Barbiturate enhancement of GABA-mediated inhibition and activation of chloride ion conductance: Correlation with anticonvulsant and anesthetic actions. Brain Res. 191: 225-237.

Schwartz, R. D., M. J. Wess, R. Labarca, P. Skolnick, and S. M. Paul (1987) Acute stress enhances the activity of the GABA receptorgated chloride ion channel in brain. Brain Res. 411: 151-155.

Scollo-Lavizzari, G., and E. Steinmann (1985) Reversal of hepatic coma by benzodiazepine antagonist (Ro 15-1788). Lancet $1: 1324$.

Sherlock, S. (1981) Hepatic encephalopathy. In Diseases of the Liver and Biliary System, pp. 91-196, Blackwell Scientific, Boston, MA.

Skerrit, J. H., and R. L. MacDonald (1983) The benzodiazepine Ro 15-1788: Electrophysiological evidence for partial agonist activity. Neurosci. Lett. 43: 321-326.

Skolnick, P., and S. M. Paul (1982) Benzodiazepine receptors in the central nervous system. Int. Rev. Neurobiol. 23: 103-140.

Skolnick, P., P. J. Marangos, F. K. Goodwin, M. Edwards, and S. M. Paul (1978) Identification of inosine and hypoxanthine as endogenous inhibitors of $\left[{ }^{3} \mathrm{H}\right]$ diazepam binding in the CNS. Life Sci. 23: 1473-1480.

Study, R. E., and J. L. Barker (1981) Diazepam and (-)-pentobarbital: Fluctuation analysis reveals different mechanisms for potentiation of GABA responses in cultured central neurons. Proc. Natl. Acad. Sci. USA 78: 7180-7184.

Tallarida, R. J., and R. B. Murray (1987) Manual of Pharmacologic Calculations with Computer Programs, pp. 153-159, Springer-Verlag, New York.

Tallman, J. F., S. M. Paul, P. Skolnick, and D. W. Gallager (1980) Receptors for the age of anxiety: The pharmacology of benzodiazepines. Science 207: 274-279.

Trullas, R., H. Havoundjian, N. Zamir, S. Paul, and P. Skolnick (1987) Environmentally-induced modification of the benzodiazepine/GABA receptor coupled chloride ionophore. Psychopharmacology 91:384390.

van Berlo, C. L. H., H. R. deJonge, A. E. J. M. van den Bogaard, M. A. Janssen, H. M. H. van Eijk, M. A. H. vander Heijden, and P. B. Soeters (1987) Gamma aminobutytric acid production in small and large intestine of conventional and germ free Wistar rats: Influence of food intake and intestinal flora. Gastroenterology 93: 472-479.

Vicini, S., H. Alho, E. Costa, J.-M. Mienville, M. R. Santi, and F. M. Vaccarino (1986) Modulation of GABA-mediated inhibitory synaptic currents in dissociated cortical cell cultures. Proc. Natl. Acad. Sci. USA 83: 9269-9273.

Wilson, M. A., and D. W. Gallager (1987) Effects of chronic diazepam exposure on GABA sensitivity and on benzodiazepine potentiation of GABA-mediated responses of substantia nigra pars reticulata neurons of rats. Eur. J. Pharmacol. 136: 333-343.

Zieve, L. (1981) The mechanism of hepatic coma. Hepatology 1: 360365.

Zieve, L. (1982) Hepatic encephalopathy. In Diseases of the Liver, L. Schiff and E. R. Schiff, eds., pp. 433-460, Lippincott, Philadelphia. 